\title{
PROGENITORS OF TYPE Ib SUPERNOVAE
}

\author{
NINO PANAGIA 1 \\ Space Telescope Science Institute \\ VICTORIA G. LAIDLER ${ }^{2}$ \\ Astronomy Programs, Computer Sciences Corporation.
}

\begin{abstract}
We argue that the progenitors of type Ib supernovae are moderately massive stars $\left(\mathrm{M} \sim 7 \mathrm{M}_{\odot}\right)$ in binary systems whereas the hypothesis that they originate from very massive stars $\left(M>20 M_{\odot}\right)$ is not consistent with the observational evidence on SNIb.
\end{abstract}

\section{Introduction}

The realization that there is a separate subclass of type I supernovae ( $\mathrm{SNe}$ ) to be denoted as type Ib came after the detailed study of the SN 1983N in M83 (Panagia et al. 1990; see also Panagia 1985, Wheeler and Levreault 1985, Uomoto and Kirshner 1985). It was immediately clear that SN $1983 \mathrm{~N}$ is distinctly different from the classical variety of type I $\mathrm{SNe}$ (obviously denoted as type $\mathrm{Ia} \mathrm{SNe}$ ) in a number of important aspects. Since then about a dozen $\mathrm{SNe}$ have been classified as type $\mathrm{Ib} \mathrm{SNe}$, some newly discovered and others found just re-examining old spectra or paying due attention to the comments that the observers gave at the time when the original observations were made (e.g. Bertola 1964). In addition to the necessary condition to be called type I SNe, i.e. the absence of hydrogen lines from the spectrum, the distinctive characteristics of type Ib SNe can be summarized as follows (cf. Panagia et al. 1986, Weiler and Sramek 1988):

- The $6150 \AA$ feature is absent from the spectrum.

- The overall spectral distribution is redder $(\Delta(\mathrm{B}-\mathrm{V}) \sim 0.5)$ and fainter $(\sim 1.5$ magnitudes) than for type Ia SNe.

- The optical light curve is essentially "normal", i.e. quite similar to that of type Ia SNe.

- The IR light curve is single-peaked, the maximum occurring a few days after the optical maximum.

\footnotetext{
${ }^{1}$ Affiliated to the Astrophysics Division, Space Science Department of ESA; on leave from University of Catania.

${ }^{2}$ Staff member, Space Telescope Science Institute.
} 
- They are strong radio emitters with a steep spectrum and a quick temporal decline.

- They are found only in spiral galaxies.

- They are located in spiral arms and are often projected near an HII region.

In the following we discuss these points in some detail and draw conclusions about the nature of SNIb progenitors.

\section{Discussion}

\subsection{OPTICAL EMISSION}

The first four points imply that SNIb originate from rather compact stars (pre-supernova radius less that $f e w \times 10^{12} \mathrm{~cm}$, hence not a red supergiant) and have an envelope possibly as small as that of type Ia SNe (because of the similarity of the light curves), but have a chemical composition different from that of type Ia progenitors (absence of the $6150 \AA$ band) and a lower amount of ${ }^{56} \mathrm{Ni}$ synthetized in the explosion (redder and fainter emission).

\subsection{RADIO EMISSION}

The substantial radio emission of SNIb requires the presence of circumstellar material, that results from pre-supernova mass loss with $\dot{M} / v_{\text {exp }} \sim 3 \times 10^{-7}\left[M_{\odot} y r^{-1}\right] /\left[\mathrm{km} \mathrm{s}^{-1}\right]$ (Weiler et al. 1986). Such a flow is rather opaque and may create a pseudo-photosphere at the base of the wind itself. For example, assuming a constant wind velocity, the radius at which the optical depth is of the order of unity is given by (Panagia and Felli 1982):

$$
R \approx 3 \times 10^{12}\left(\kappa / \sigma_{e}\right)\left\{\left(\dot{M} / v_{\text {exp }}\right) / 10^{-7}\right\}[\mathrm{cm}]>9 \times 10^{12}[\mathrm{~cm}]
$$

where $\kappa$ is the average opacity, $\sigma_{e}$ is the electron scattering cross section, $\dot{M}$ is in $M_{\odot} y r^{-1}$ and $v_{\exp }$ in $\mathrm{km} \mathrm{s}^{-1}$. A radius like this is about an order of magnitude larger than that of a WR star and is too large for the SNIb progenitor because it would produce a light curve much broader than observed. On the other hand, the value of $\dot{M} / v_{\text {exp }} \sim$ $3 \times 10^{-7}\left[M_{\odot} y^{-1}\right] /\left[k^{-1}\right]$ is appropriate for a red supergiant. Therefore, the SNIb progenitor has to have a relatively massive companion (i.e. several solar masses; Sramek et al. 1984) that at the time of the explosion is in the red supergiant phase. Note that the binary system hypothesis to explain the radio emission of SNIb is not applicable to the case of a WR progenitor because the WR wind would completely sweep out the wind of a possible red supergiant companion and, again, the density in the circumstellar material would be far too low to produce the radio phenomenon (i.e. to accelerate the relativistic electrons that emit synchrotron radiation and to cause the $f-f$ absorption that delays the rise of the flux at long wavelengths).

\subsection{LOCATION IN THE GALAXY}

In order to satisfy the condition of being located in spiral arms, the lifetime of SNIb progenitors must be shorter than $3 \times 10^{8}$ years, and, therefore their original mass larger than 5.5 $\mathrm{M}_{\odot}$. In fact, if type Ib SNe were intrinsically associated with HII regions, their progenitors 
may be short-lived and, therefore, be appreciably more massive.

Wheeler et al. (1987) have claimed that about $50 \%$ of type Ib SNe are associated with HII regions. We have made a systematic study of the separations of SNe from HII regions in spiral galaxies by overlaying the best positions of $\mathrm{SNe}$ [among those reported in the Barbon et al. Catalog of $\mathrm{SNe}[12]$ and those astrometrically determined either in the optical or in the radio] on the galaxy images with the use of the GASP ${ }^{3}$ software (Panagia and Laidler 1990. For type Ib SNe we find that 6 out of 11 appear to fall within 5" from the center of a knot (i.e. presumably an HII region).

Actually, such an "association" with an HII region actually means close proximity because the median absolute distance of SNIb from HII regions turns out to be about 200 pc: this is much larger than the distance of early $O$ type stars from giant HII regions in our Galaxy. On the other hand, a similar distribution of separations from the nearest HII region is found for SNII in spiral galaxies while for SNIa the distances from HII regions are much larger (the median distance is about $600 \mathrm{pc}$ ). This confirms the idea that the progenitors of both SNIb and SNII belong to a young stellar population but is not enough to derive quantitative estimates of lifetimes and/or masses of the progenitors. The main problems here are the limited sample, the poor resolution of ground based observations (we remind that for a galaxy at $10 \mathrm{Mpc}$ a separation of 1" corresponds to a distance of $50 \mathrm{pc}$ !), and insufficient accuracy of recorded positions of SNe (typically affected by errors of 3" or more). These last two effects may be responsible of increasing the dispersion and make the apparent correlation less tight than it may be in reality.

In any case, it is clear that SNIb cannot be the result of the explosion of a WR star because the strong radio emission at early epochs cannot be explained with the presence of a WR star. Therefore, the progenitors of type Ib SNe are likely to be only moderately massive stars, i.e. in the low part of the mass distribution of massive stars, say, around 10 $\mathrm{M}_{\odot}$.

\subsection{SNIb STATISTICS}

The range of possible progenitor's masses can be estimated considering that the frequency of type Ib explosions in spiral galaxies is about $1 / 3$ that of type II SNe (Branch 1986, van den Bergh, McClure, and Evans 1987). Therefore, assuming that stars more massive than $8 \mathrm{M}_{\odot}$ make type II SNe (e.g. Maeder 1987) and adopting an initial mass function proportional to $M^{-2.35}$ the possible mass range for $\mathrm{SN} \mathrm{Ib}$ progenitors turns out to be about 6.5-8 $\mathrm{M}_{\odot}$. This agrees well with the direct estimate of $\mathrm{M}>6.5 \mathrm{M}_{\odot}$ made by Sramek et al. (1984) for SN 1983N on the basis of its radio emission. Also, a relatively "modest" mass for the progenitor can naturally explain why the mass ejected in the explosion (as implied by the "normal" optical light curve) is a few solar masses at most (Branch 1988).

\section{Conclusions}

We conclude that the only viable scenario to account for type Ib events is that of a star with original mass around $7 \mathrm{M}_{\odot}$, which is member of a binary system in which the companion is slightly less massive (say, $\sim 5 \mathrm{M}_{\odot}$ ). The primary follows its evolution to the end becoming a rather massive degenerate star, which explodes when the secondary has reached the stage

\footnotetext{
${ }^{3}$ GASP is the Guide Star Astrometric Support Program available at the Space Telescope Science Institute.
} 
of red supergiant. The alternative hypothesis of a very massive progenitor (i.e. $M>20-30$ $\mathrm{M}_{\odot}$ ) is ruled out on the basis of the mass loss characteristics required to account for the radio emission and by the low mass envelope implied by the behaviour of the optical and UV light curves.

Finally, let us stress that our point is not that WR stars do not make SN explosions but only that they do not make SNIb events. For example, we wonder if the core collapse of a WR may be "gentle" enough to produce "dim" explosions such as that believed to have given rise to Cas $\mathrm{A}$.

\section{References}

Barbon, R., Cappellaro, E., Ciatti, F., Turatto, M., Kowal, C.T., 1984, Astron. Astrophys. Suppl., 58, 735.

Bertola, F., 1964, Ann. Astrophys., 27, 319.

Branch, D., 1986, Astrophys. J. (Letters), 300, L51.

Branch, D., 1988, IAU Colloquium No. 108, ed. K. Nomoto (Berlin: Springer Verlag), p. 281.

Maeder, A., 1987, ESO Workshop "SN 1987A", ed. I.J. Danziger, p. 251.

Panagia, N., 1985, in "Supernovae as Distance Indicators", ed. N. Bartel (Berlin: Springer), p. 14.

Panagia, N., Felli, M.: in "Wolf-Rayet Stars: Observations, Physics, Evolution", eds. C.W.H. de Loore and A.J. Willis (Dordrecht: Reidel), p. 203 (1982).

Panagia, N., Laidler, V.G., 1990, Astrophys. J., in press.

Panagia, N., Sramek, R.A., Weiler, K.W., 1986, Astrophys. J. (Letters), 300, L55.

Panagia, N. et al, 1990, in preparation.

Sramek, R.A., Panagia, N., Weiler, K.W., 1984, Astrophys. J. (Letters), 285, L59.

Uomoto, A., Kirshner, R.P., 1985, Astron. Astrophys., 149, L7.

van den Bergh, S., McClure, R.D., Evans, R., 1987, Astrophys. J., 323, 44.

Weiler, K.W., Sramek, R.A., Panagia, N., van der Hulst, J.M., Salvati, M., 1986, Astrophys. J., 301, 790.

Weiler, K.W., Sramek, R.A., 1988, Ann. Rev. Astron. Astrophys., 26, 295.

Wheeler, J.C., Levreault, R., 1985, Astrophys. J. (Letters), 294, 17.

Wheeler, J.C., Harkness, R.P., Cappellaro, E., 1987, Proc. 13th Texas Symposium on Relativistic Astrophysics, ed. M.P. Ulmer (Singapore: World Scientific), p. 402. 